\title{
Splenic Infarct: A Rare Presentation in a Pediatric Patient
}

\author{
Pallav Bhattarai,' Louidson Pierr, 'Adebayo Adeyinka, 'Swayam Sadanandan' \\ 'The Brooklyn Hospital Center, Brooklyn NY, The United States of America.
}

\section{ABSTRACT}

A previously healthy 16 -year-old male presented with a two day history of persistent epigastric pain. His physical examination was significant for tenderness in the left hypochondriac region with a palpable spleen $2 \mathrm{~cm}$ below the left sub-costal margin. A CT scan of the abdomen showed a splenic infarct. Heterophile and EBV VCA IgM antibody test were positive. This is a rare case of infectious mononucleosis presenting with splenic infarct in an adolescent male without comorbidities.

Keywords: infectious mononucleosis; splenic infarct.

\section{INTRODUCTION}

Several mechanisms have been proposed to explain the underlying pathophysiology of splenic infarction associated with infectious mononucleosis. These include both hypoxemia and increased thrombotic tendency due to underlying conditions. Acute EBV infection also induces excess $B$ cell proliferation and consequently, increased production of various antibodies. It has been shown that EBV also leads to transient increase in thrombotic markers. Beyond these hypotheses, specific pathophysiologic mechanisms leading to splenic infarct need further investigation.

The clinical presentation of infarction may include signs and symptoms characteristic of hemorrhagic shock or septic thromboembolism which often results in splenic abscesses. Moreover, classic signs of severe sepsis with left upper abdominal pain are typical. ${ }^{1}$ CT scan is the current diagnostic modality of choice because there are no known specific laboratory tests to make an accurate diagnosis. CT scan with gadolinium contrast and three dimensional reconstruction can be used for better image enhancement. ${ }^{2-4}$ Subcapsular hematoma evident on the CT scan may be a sign of splenic rupture.
We present a case of a 16 -year-old male with EBV positive infectious mononucleosis associated with splenic infarction as a sequela. The patient was managed conservatively with subsequent resolution of clinical symptoms.

\section{CASE REPORT}

A previously healthy 16 -year-old male presented with a two day history of persistent epigastric pain worsening on inspiration and improving at rest. He also reported one episode of non-bilious, non-bloody emesis. The patient denied fever, headache, sore throat, myalgia, weight loss and arthralgia. He was visiting the United States from the Dominican Republic. He also denied any history of trauma, recent URI or previous episodes of similar symptoms.

Correspondence: Dr. Pallav Bhattarai, The Brooklyn Hospital Center, Brooklyn; NY; The United States of America. Email: pallavorama@gmail.com. 
There was no family history of any known illnesses. On physical examination, there was no erythema or exudates of the pharyngeal mucosa and there was no cervical lymphadenopathy. There were no bruises or petechiae. Abdominal exam was significant for tenderness in the epigastrium with increased tenderness in the left hypochondriac region. The splenic tip was palpable $2 \mathrm{~cm}$ below the left sub-costal margin in the splenic axis. Cardiovascular, respiratory, musculoskeletal and neurological exams were unremarkable.

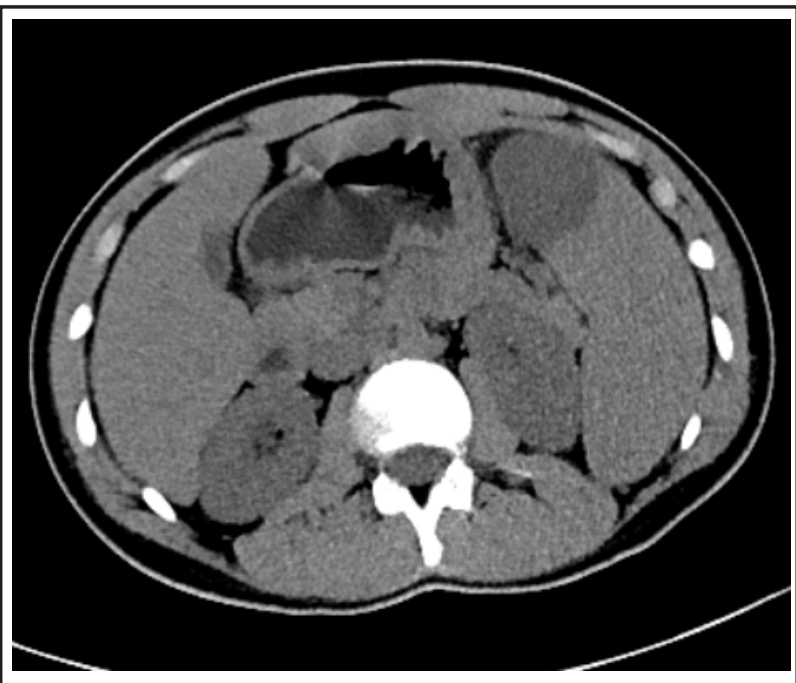

Figure 1.CT scan showing mild hepato-splenomegaly with a $4.3 \mathrm{~cm}$ sub-capsular low-attenuation area without capsule destruction consistent with infarction.

\section{INVESTIGATIONS}

The $\mathrm{CBC}$ on admission showed $\mathrm{Hb}$ of $12.3 \mathrm{~g} / \mathrm{dl}$ and Hct of $36.2 \%, \mathrm{MCHC}$ of $35.2 \mathrm{~g} / \mathrm{dl}, \mathrm{WBC}$ of 6.76 with $11 \%$ Atypical Lymphocytes. Other labs included ALT/AST: 154/105 U/L, PT: $16.7 \mathrm{Sec}$, INR: 1.53, PTT: $35 \mathrm{Sec}$, Total bilirubin: $1.5 \mathrm{mg} / \mathrm{dl}$, LDH $552 \mathrm{U} / \mathrm{L}$ and Uric Acid 4.8 $\mathrm{mg} / \mathrm{dl}$. The peripheral smear was normal. CT scan of the abdomen showed mild hepato-splenomegaly with a 4.3 $\mathrm{cm}$ sub-capsular low-attenuation area without capsule destruction consistent with infarction. Heterophile antibody test and EBV VCA IgM were positive thus confirming acute infectious mononucleosis with splenic infarct.

\section{OUTCOME AND FOLLOW UP}

The patient symptomatically improved over the following two days with conservative management. The laboratory tests were repeated and showed normalization of PT/ INR (13.9/1.3) as well as a downward trend in AST: 74, ALT: 124, Total bilirubin: 1.3, LDH 486. He was discharged with instructions to avoid contact sports for three weeks and to follow up with his primary care physician in the Dominican Republic.

\section{DISCUSSION}

Splenic infarct in children is a rare finding without preexisting hematological abnormalities. During history taking or clinical examination, hematological or other etiologies are mostly identified. In this case, the other causes were excluded based on history, physical examination and peripheral blood smear. In some patients when specific risk factors are not identified, an uncommon underlying etiology is uncovered. Splenic infarction with EBV positive mononucleosis without comorbidities is exceedingly rare. The associated comorbidities include hereditary spherocytosis, protein C deficiency, ${ }^{5}$ sickle cell disease ${ }^{6}$ and pyruvate kinase deficiency. ${ }^{7}$ EBV has been seen to cause transient elevated antiphospholipid antibodies and in one case resulted in infarction. ${ }^{8}$ When comorbid disorders are excluded, splenic infarctions with infectious mononucleosis have only been reported three times. ${ }^{9,10}$ Since the diagnosis of infectious mononucleosis is usually made clinically, CT scans are not routinely performed and the incidence of splenic infarction due to infectious mononucleosis cannot be determined. ${ }^{11}$ In children surgery is indicated only in the presence of complications with hypersplenism or focal splenic tumors. ${ }^{12}$

\section{REFERENCES}

1. Guth AA, Pachter HL. Splenic infarct. e Medicine J. 2002;3(4):1-11.

2. Balcar I, Seltzer SE, Davis S. CT patterns of splenic infarction: a clinical and experimental study. Radiology. 1984;151(3):723-9.

3. Cohen BA, Mitrty HA, Mendelson DS. Computed tomography of splenic infarction. J Comput Assist Tomogr. 1984;8(1):167-8.
4. Kluger Y, Paul DB, Townsend RN. Enhanced rim around infarcted, traumatised spleen on computed tomographic scans: Case report. J Trauma. 1994;36(3):436-7.

5. Breuer C, Janssen G, Laws HJ, Schaper J, Mayatepek E, Schroten H, et al. European Journal Of Pediatrics. 2008 Dec;167(12):1449-52. 
6. Symeonidis A, Papakonstantinou C, Seimeni U, Sougleri M, Kouraklis-Symeonidis A, Lambropoulou-Karatza C, et al. Non Hypoxia-Related Splenic Infarct in a Patient with Sickle Cell Trait and Infectious Mononucleosis. Acta Haematol. 2001;105(1):53-6.

7. Boivin P, Bernard JF. Deficit en pyruvate kinase, mononucleose infectieuse, anemie hemolytique a autoanticorps froids et infarctus splenique massif. Presse Medicale. 1990 Apr;19(17):818-9.

8. Van Hal S, Senanayake S, Hardiman R. Splenic infarction due to transient antiphospholipid antibodies induced by acute Epstein-Barr virus infection. J Clin Virol. 2005 Mar;32(3):245-7.
9. Gavriilaki E1, Sabanis N, Paschou E, Grigoriadis S, Mainou M, Gaitanaki A, et al. Splenic infarction as a rare complication of infectious mononucleosis due to Epstein-Barr virus infection in a patient with no significant comorbidity: case report and review of the literature. Scand J Infect Dis. 2013 Nov;45(11):888-90.

10. Trevenzoli M, Sattin A, Sgarabotto D, Francavilla E, Cattelan AM. Splenic infarct during infectious mononucleosis. Scand J Infect Dis. 2001;33(7):550-1.

11. Garten AJ, Mendelson DS, Halton KP. CT manifestations of infectious mononucleosis. Clin Imaging. 1992 Apr-Jun;16(2):114-6.

12. Hery G, Becmeur F, Mefat L, Kalfa D, Lutz P, Lutz L, et al. Laparoscopic partial splenectomy: indications and results of amulticenter retrospective study. Surg Endosc. 2008 Jan;22(1):45-9. 